\title{
Carbon dioxide transoral laser microsurgery - the gold standard for early glottic laryngeal carcinomas?
}

\author{
Nezi Nizamova \\ Department of ENT Surgery \\ University Hospital "Queen Giovanna" - ISUL \\ Medical University - Sofia
}

\begin{abstract}
Early glottic squamous cell carcinoma (Tis-T1) is a highly treatable disease by any of the main treatment methods available. Carbon dioxide transoral laser microsurgery (TLM) and radiotherapy (RT) are considered the two main treatment methods for early glottic carcinomas. However, the both treatment strategies have advantages and disadvantages. TLM is by many authors described as the gold standard mainly due to better overall survival, shorter hospitalization, lower morbidity, and arguably better functional outcomes compared to those of RT. Furthermore, TLM as a primary treatment strategy leaves open all treatment options in patients with recurrent disease.
\end{abstract}

This work is supported by the Bulgarian Ministry of Education and Science under the National Program for Research "Young Scientists and Postdoctoral Students"

\section{Introduction:}

\section{Brief History of CO2 Laser of the Larynx}

The development of new equipment and techniques inspired Prof. Geza Jako to apply the CO2 laser energy in the larynx of cadavers at the beginning of the 1970s. This led to publishing a study about the use of a carbon dioxide laser in the larynx of 13 dogs in 1972 [1].

In the same year, Strong and Jako were the first to publish the use of $\mathrm{CO} 2$ laser, in combination with microscopic visualization and direct laryngoscopy, for the surgical treatment of benign laryngeal lesions in human patients [2]. Annyas, Steiner, Eckel are well know as they all have contributed to the beginning of the spread of the technique across the continent [3].

Laryngeal cancer is the second most common cancer in the upper aerodigestive tract. Approximately $95 \%$ of laryngeal cancers are squamous cell carcinomas (SCC), and a significant majority occur in patients between 60 and 70 years old [4].

Early cancer of the glottis refers to a cancer that has not spread to adjacent spaces of the larynx and corresponds to the stages Tis, T1, and T2 (AJCC/ UICC TNM, 8th edition) [5].

Patients present with hoarseness as the first symptom of cancer of glottis. The early diagnosis is quite relevant, since advanced lesions require more aggressive treatment modalities.

The extent of the neoplasm can be evaluated by using imaging examinations, such as computed 
tomography (CT) and magnetic resonance imaging (MRI), particularly in cases in which all limits cannot be determined with direct laryngoscopy. However, laryngoscopy remains the most important examination for diagnosing and staging of early cancer of the glottis [3].

Treatment of early stage laryngeal cancer developed over the last five decades especially with the transition from the classic open surgical resection methods to the less aggressive and more functional endoscopic approach. Nowadays treatment options show good local control, high laryngectomy-free survival rates and overall high success rates $80 \%-$ 90\%. [6]

An important goal of initial treatment of early glottic squamous cell carcinoma (Tis-T2), other than survival, is functional organ preservation by achieving local control while avoiding total laryngectomy. When choosing treatment strategies, the secondary parameters such as functional outcome (voice, swallowing etc.), morbidity, and quality of life also play a large role. Different types of treatment modalities, such as transoral laser microsurgery (TLM), radiotherapy (RT), or open partial laryngectomy (OPL) can be considered for treatment options [7].

Series of studies revealed best results with TLM because of its low management costs, reduced hospitalization time, and good oncological and functional results, with low complication rates and less severe complications when compared to the other techniques $[8,9]$.

RT offers similar oncological and functional outcomes, but higher costs, prolonged time of treatment, mucosal damage, and long-term side effects such as xerostomia [10].

\section{Discussion}

Initial treatment of early glottic squamous cell carcinoma aims at various goals as overall survival, laryngeal preservation by achieving local control while avoiding total laryngectomy.

Both TLM and RT offer similar outcomes as treatment options for early glottic carcinomas. The selection of treatment strategy depends not only overall survival rate but on functional outcomes as voice, swallowing and quality of life. Due to the lack of randomized controlled trials accessing this issue, it is hard to say which of the therapeutic options gives better outcomes.

\section{Overall survival and laryngeal preservation.}

A recent meta-analysis from 2019, published in Journal of Otolaryngology - Head and Neck Surgery, included 1987 patients from 16 cohort studies. Vaculic et al. demonstrated that TLM was associated with better outcomes in terms of overall survival, disease-specific survival and laryngeal preservation [11].

Mo and co-authors published a meta-analysis in 2016, which showed significantly improved overall survival of patients with $\mathrm{T} 1$ glottic carcinoma in the TLM ( $p=0.04)$ and significantly better laryngeal preservation. As a limitation of the study authors point to the fact, that some studies did not have clear indications for inclusion of patients in TLM or RT treatment [12].

Gioacchini and co-authors concluded that there are no significant differences in terms of survival rate in both groups [13].

An older meta-analysis from 2009, which is based on over 7600 patients, favors the TLM in comparison to RT treatment for overall survival. Higgins and co-authors did not find no differences in local control and laryngectomy-free survival as well as for the voice [14].

\section{Fuctional outcomes - voice and quality of life}

Greulich et al. published a meta-analysis in 2015, which included 207 patients receiving RT and 155 patients with receiving TLM from 8 research papers T1 glottic carcinoma. The meta-analysis focused on VHI (Voice Handicapped Index), which evaluates the voice outcomes after RT and TLM treatment. There was no statistical significant difference between subjective voice outcomes following XRT versus surgery for treatment of T1 glottic carcinoma. [15]

Guangyuan Du et al. punished a meta-analysis also in 2015, which included 368 patients receiving RT and 440 patients with receiving TLM from 13 articles. The meta-analysis focused on VHI (Voice Handicapped Index), which evaluates the voice outcomes after RT and TLM treatment. Authors investigated the acoustic voice analyses, which included fundamental frequency, shimmer and jitter. Authors did not registered any differences in the jitter and shimmer qualities, but indicated that TLMR has advantage in the fundamental frequency 
in comparison to the RT [16].

Lee and co-authors published a meta-analysis of acoustic parameters in 744 patients with early glottic carcinoma (T1 and T2), which showed significant differences in jitter, shimmer, noise to harmonic ratio with more favorable values in the RT group than those in the TLMS group [17].

\section{Conclussion}

RT and TLM are two viable options for treatment of early glottic cancer. Both have their advantages and limitations. Although TLM seems to have an advantage in comparison to RT therapy in patients with $\mathrm{T} 1$ carcinoma, management of those patients should be individualized, according to surgical risk factors, advanced stage, deeper layers, or in cases of refusal of surgical intervention. Prospectively randomized controlled, multicenter studies are required to further prove the efficacy of initial TLM over initial RT therapy.

\title{
References:
}

1. Jako, G.J. Laser surgery of the vocal cords. An experimental study with carbon dioxide lasers on dogs. Laryngoscope 1972, 12, 2204-2216.

2. Strong, M.S.; Jako, G.J. Laser surgery in the larynx. Early clinical experience with continuous CO2 laser. Ann. Otol. Rhinol. Laryngol. 1972, 81, 791-798.

3. Chiesa-Estomba CM, González-García JA, Larruscain E, Calvo-Henríquez C, Mayo-Yáñez M, Sistiaga-Suarez JA. CO Transoral Laser Microsurgery in Benign, Premalignant and Malignant (Tis, T1, T2) Lesion of the Glottis. A Literature Review. Medicines (Basel). 2019 Jul 22;6(3):77.

4. Barnes L, Tse LLY, Hunt JL, Brandwein-Gensler M, Urken M, Slootweg P, Gale N, Cardesa A, Zidar N, Boffetta P (2005) Tumours of the hypopharynx, larynx and trachea: Introduction. In: Barnes L, Eveson JW, Reichart P, Sidransky D (eds) World Health Organization Classification of Tumours Pathology and Genetics of Head and Neck Tumours. IARC Press, Lyon, pp 111-117

5. Amin MB, Sullivan DC, Jessup JM, et al, eds. American Joint Committee on Staging Manual. 8th ed. New York, NY: Springer; 2017

6. Mendelsohn, A.H.; Remacle, M.J. Vocal Fold Cancer Transoral Laser Microsurgery Following European Laryngological Society Laser Cordectomy Classification. Front. Oncol. 2018, 8, 231.

7. Sjögren EV. Transoral Laser Microsurgery in Early Glottic Lesions. Curr Otorhinolaryngol Rep. 2017;5(1):56-68

8. Goor KM, Peeters AJ, Mahieu HF, et al. Cordectomy by $\mathrm{CO}_{2}$ laser or radiotherapy for small T1a glottic carcinomas: costs, local control, survival, quality of life, and voice quality. Head Neck. 2007;29(2):128-136.

9 Diaz-de-Cerio P, Preciado J, Santaolalla F, Sanchez-Del-Rey A. Cost-minimisation and cost-effectiveness analysis comparing transoral CO laser cordectomy, laryngofissure cordectomy and radiotherapy for the treatment of T1-T2, N0, M0 glottic carcinoma. Eur Arch Otorhinolaryngol. 2013;270(4):1181-1188

10. Mendenhall WM, Amdur RJ, Morris CG, Hinerman RW. T1-T2N0 squamous cell carcinoma of the glottis larynx treated with radiation therapy. J Clin Oncol. 2001;19(20):4029-4036.

11. Vaculik M, MacKay C, Taylor M et al. Systematic review and meta-analysis of T1 glottic cancer outcomes comparing CO2 transoral laser microsurgery and radiotherapy Vaculik et al. Journal of Otolaryngology - Head and Neck Surgery (2019) 48:44

12. Mo H, Li J, Yang X. et al. Transoral laser microsurgery versus radiotherapy for T1 glottic carcinoma: a systematic review and meta-analysis; Lasers Med Sci 2015 August;

13. Gioacchini FM, Tulli M, Kaleci S, Bondi S, Bussi M, Re M. Therapeutic modalities and oncologic outcomes in the treatment of T1b glottic squamous cell carcinoma: a systematic review. Eur Arch Otorhinolaryngol. 2017 Dec;274(12):4091-4102.

14. Higgins KM, Shah MD, Ogaick MJ, Enepekides D. Treatment of early-stage glottic cancer: meta-analysis comparison of laser excision versus radiotherapy. J Otolaryngol Head Neck Surg. 2009 Dec;38(6):603-12. PMID: 19958721.

15 Greulich M, Parker N, Lee P et al. Voice Outcomes Following Radiation Versus Laser Microsurgery for T1 Glottic Carcinoma: Systematic Review and Meta-analysis; Otolaryngol Head Neck Surg. 2015 May ; 152(5): 811-819. doi:10.1177/0194599815577103.

16. Du G, Liu C, Yu W et al. Voice outcomes after laser surgery vs. radiotherapy of early glottic carcinoma: a meta-analysis; Int J Clin Exp Med 2015;8(10):17206-17213

17. Lee S, Hong K, KimJ et al. Perceptual and Acoustic Outcomes of Early Stage Glottic Cancer After Laser Surgery or Radiotherapy: A Meta-Analysis; Clinical and Experimental Otorhinolaryngology Vol. 12, No. 3: 241-248, August 2019

\author{
Correspondence to: \\ Nezi Nizamova, MD \\ Department of ENT Surgery \\ University Hospital "Queen Giovanna" - ISUL \\ Byalo More Str 8 \\ Medical University - Sofia \\ E-mail: nezi.nizamova@gmail.com
}

